\title{
Die subtrochantäre Femurfraktur
}

\author{
Thomas Strohecker, Axel Pommer, Andreas Dávid
}

\section{Zusammenfassung}

Subtrochantäre Frakturen sind im Gegensatz zu den pertrochantären und medialen Schenkelhalsfrakturen Verletzungen, die auch bei jugendlichen Patienten gehäuft auftreten. Eine operative Versorgung dieser Brüche ist obligatorisch. Ziel ist, die Stabilität und damit die rasche Belastbarkeit des Oberschenkels zu erreichen. Für die Versorgung dieser Frakturen stehen verschiedene Platten- und intramedulläre Nagelsysteme zur Verfügung. Diese werden wegen der minimal invasiveren Zugänge und der hohen mechanischen Belastung heute favorisiert.

\section{Einleitung}

Subtrochantäre Frakturen sind im Gegensatz zu den anderen Frakturen des proximalen Femurs Verletzungen, die auch bei jugendlichen Patienten gehäuft auftreten. Während bei alten Menschen bereits banale Stürze zu diesem Bruchtyp führen können, sind subtrochantäre Frakturen bei jugendlichen Patienten meist Folge von Hochrasanztraumen und mit anderen Verletzungen kombiniert [4]. Diese Bruchform ist hochgradig instabil und kann daher nach heutigen Kriterien auch nicht konservativ behandelt werden. Jegliche Extensionstherapie müsste über 3-4 Monate konsequent mit der hierzu notwendigen Bettruhe durchgeführt werden. Zudem kann wegen des sehr starken Muskelzuges (M. psoas und $\mathrm{Mm}$. gluteus maximus und medius)

OP-JOURNAL 2002; 17: 142-146

(c) Georg Thieme Verlag Stuttgart · New York eine achsgerechte Einstellung der Fraktur allein über eine Extension kaum erreicht werden.

\section{Einteilung}

Bei subtrochantären Frakturen werden unterschiedliche Klassifikationen angewandt. Die Fraktur liegt unterhalb des Trochanter minor und wird damit von den pertrochantären Frakturen eindeutig abgegrenzt. Die distale Begrenzung der subtrochantären Region ist dagegen nicht eindeutig definiert. Sehr weit verbreitet ist die Beschreibung der Frakturen in Anlehnung an die AO-Klassifikation für die diaphysären Frakturen.

\section{Klinik}

Die Frakturzeichen sind in der Regel eindeutig. Heftigste Schmerzen im Bereich des proximalen Oberschenkels, starke Schwellung und eine Fehlstellung mit Verkürzung des Oberschenkels deuten auf den Frakturtyp hin.

\section{Präoperative Diagnostik und Vorbereitung}

Bei klinischer Verdachtsdiagnose einer subtrochantären Fraktur sollte nach Prüfung der Sensibilität, Motorik und Durchblutung die Röntgendiagnostik eingeleitet werden. Sinnvoll ist es, Patienten mit instabilen Frakturen und starken Schmerzen auf eine Vakuummatratze zu lagern und die weitere Diagnostik auf dieser Matratze fortzuführen. Andere Schienungstechniken sind ineffektiv.

Die Röntgendiagnostik sollte neben einer tief eingestellten Beckenübersichtsaufnahme auch das betroffene Hüftgelenk in zwei Ebenen umfassen. Aufnahmen von Knie und distalem Femur sind nötig, um Frakturausläufer bis in diesen Bereich nicht zu übersehen, die Konsequenzen für die Implantatwahl hätten. Bei den geriatrischen Patienten ist in der Regel zur Narkosevorbereitung eine Thoraxüber- sicht erforderlich. Auf zusätzliche Schenkelhalsfrakturen muss geachtet werden!

Anamnestisch sollten die Vorerkrankungen sowie die aktuelle medikamentöse Therapie eruiert werden. Eine länger dauernde Antikoagulantientherapie oder eine dekompensierte Herzinsuffizienz kann den OP-Zeitpunkt hinauszögern. Wegen der Schwere der Fraktur und der hohen Instabilität sollte durch eine entsprechende Intensivtherapie ein zügiger Operationstermin erzwungen werden.

Bei der Versorgung der subtrochantären Frakturen - unabhängig von der Implantatwahl - ist mit einem hohen Blutverlust zu rechnen. Daher sollten Blutkonserven bereitgestellt werden. Zur peri- und postoperativen Bilanzierung ist ein Blasenverweilkatheter sinnvoll. Eine Thromboseprophylaxe ist wegen des hohen Thromboserisikos dieser Verletzungen immer erforderlich.

\section{Lagerung}

Grundsätzlich sollte die Lagerung eine Durchleuchtung des Femurs und des Hüftgelenkes ermöglichen. Günstig ist hierbei die Rückenlagerung mit Auslagerung des nicht betroffenen Beines in einer Beinschale. Eine reine Seitenlagerung bewährt sich bei rein subtrochantären Frakturen nicht. Alternativ kann ein Extensionstisch verwendet werden. Die intraoperative geschlossene Reposition kann aber erschwert werden, wenn die Fraktur bodenwärts durchhängt. Wir verwenden bei der Versorgung dieses Frakturtypes in unserer Klinik keinen Extensionstisch (Abb.1a u.b).

\section{Implantatwahl}

Für die Versorgung subtrochantärer Femurfrakturen konkurrieren extramedulläre Kraftträger mit intramedullären Implantaten. Während in den vergangenen Jahrzehnten nur extramedulläre Verfah- 


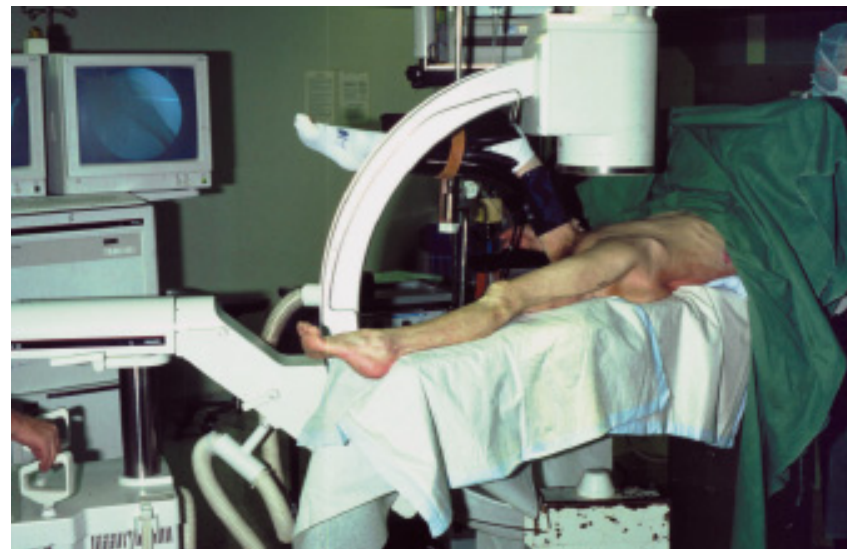

Abb.1a Lagerung der Patienten.

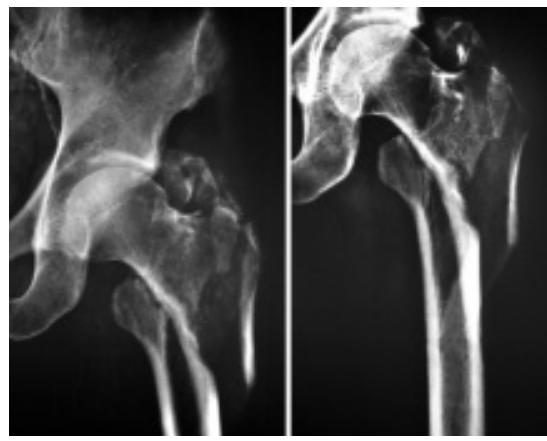

Abb. 2a Subtrochantäre Fraktur.

ren zur Verfügung standen, hat in den letzten Jahren eine Abkehr von diesen nicht primär belastungsstabilen Implantaten hin zu mehr „biologischen“ Osteosynthesen mit intramedullären Kraftträgern stattgefunden. Im Folgenden werden die gängigsten Verfahren mit ihren Vorteilen und Problemen dargestellt.

\section{Kondylenplatte}

Bei der Verwendung der $95^{\circ}$-Kondylenplatte wird eine Klinge in den Schenkelhals eingeschlagen und durch Anlage der Platte auf der Zuggurtungsseite des Femurs die Fraktur reponiert. Sie kann nur bei intaktem Trochantermassiv angewandt werden.

Die Implantation kann in Anlehnung an die konventionelle Osteosynthesetechnik mit offener anatomisch exakter Reposition (Abb. 2 a u.b) und als Überbrückungsplatte erfolgen. Diese Technik führt zu einer schnelleren Bruchheilung und sollte daher heute bevorzugt angewandt werden. Aber auch bei Implantation in durchgeschobener „biologischer“ Technik sind im Vergleich zu den intramedul-

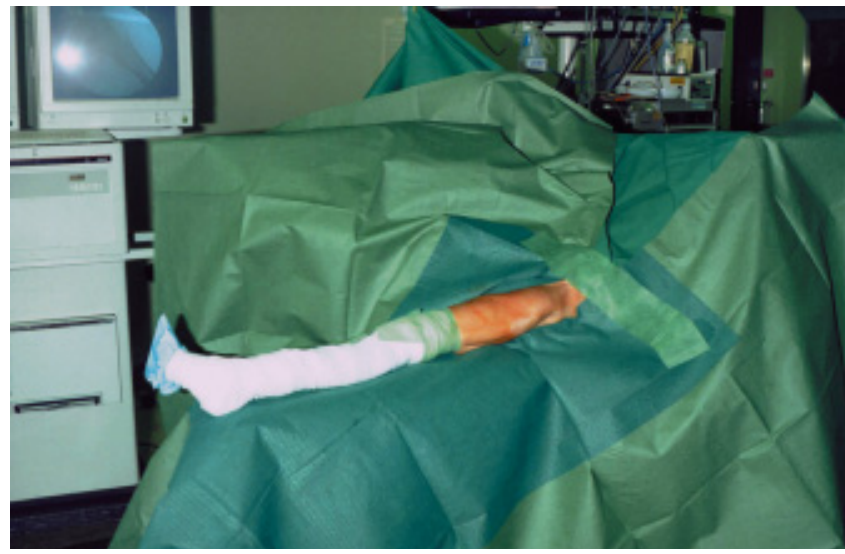

Abb.1b Abdeckung. Die Bildwandlerposition sollte eine Darstellung des proximalen Femurs auch im axialen Strahlengang zulassen.

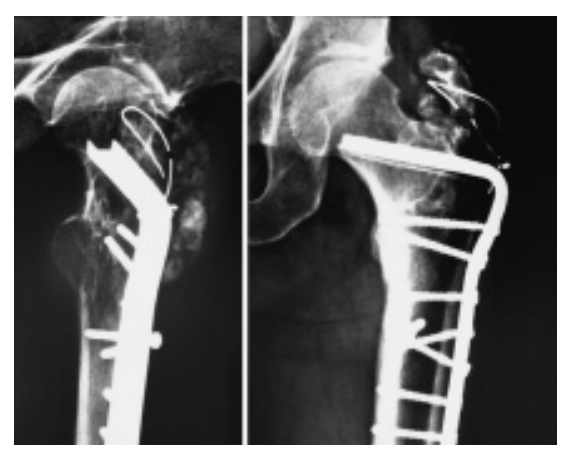

Abb. 2b Konventionelle Versorgungstechnik mit $95^{\circ}$-Kondylenplatte. Anatomische Reposition.

lären Implantaten oft Freilegungen der Bruchzone erforderlich, um eine Reposition der stark verschobenen Bruchenden zu erreichen. Die konventionelle Technik erfordert zumeist eine Spongiosaplastik an der medialen Seite der Fraktur.

Es handelt sich um ein technisch schwieriges Verfahren, das in der Hand des Ungeübten viele Fehlermöglichkeiten mit sich bringt. Durch Fehllage der Klinge im Schenkelhals kann die mediale oder dorsale Kortikalis verletzt werden. Durch die vorgegebene Form der Platte resultiert außerdem eine ungenügende Reposition der Fraktur. Nach Einschlagen der Klinge kann auch die Anpassung der Platte an den Femurschaft problematisch sein, da die Kondylenplatte im Gegensatz zur dynamischen Kondylenschraube keine Rotation um die Klinge erlaubt. Damit kann die Platte entweder ventral oder dorsal des Femurschaftes zu liegen kommen. Da in den meisten Fällen eine mediale Trümmerzone besteht und somit auf der Seite der größten Kompressionskräfte die Abstützung fehlt, besteht trotz des ausgedehnten Zugangs und den damit eingegangenen Risiken fast nie Belas-

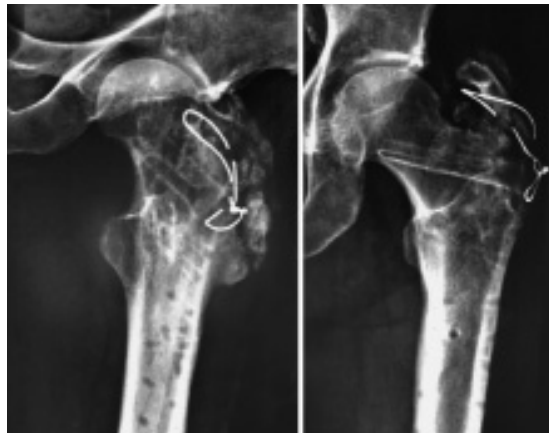

Abb. 2c Nach ME.

tungsstabilität. Dies ist der Grund, warum die $95^{\circ}$-Klingenplatte bei der Versorgung der frischen subtrochantären Fraktur seltener angewandt wird.

\section{Dynamische Kondylenschraube (DCS)}

Wie bei der Kondylenplatte handelt es sich bei der DCS um eine Platte, die an der lateralen Zuggurtungsseite des Femurs angebracht wird. Die proximale Befestigung erfolgt durch eine horizontal in den Schenkelhals eingebrachte Gleitschraube. Wie bei der Kondylenplatte ist eine intakte Trochanterregion erforderlich. Das Implantat eignet sich daher nicht für bis in die Trochanterregion reichende Frakturen. Durch das Implantat ist bei subtrochantären Frakturen eine exakte Rekonstruktion der Beinachse möglich, vor allem wenn die anatomisch exakte Einrichtung des Bruches durchgeführt wird. Allerdings ist diese Technik zugunsten der biologischen Überbrückungsplatten heute weitgehend verlassen worden.

Die DCS hat gegenüber der Kondylenplatte einige wichtige Vorteile. 


\section{Vorteile der DCS gegenüber der Kondylenplatte}

Die Steifigkeit der DCS sowie die Winkelstabilität ist gegenüber der Kondylenplatte deutlich erhöht. Nach Einbringen der Schraube ist eine Korrektur der Plattenlage um die Schraubenachse möglich. Nach Einschlagen der Klinge kann die $95^{\circ}$-Kondylenplatte entlang des $\mathrm{Fe}$ murschaftes nicht mehr korrigiert werden

Durch das getrennte Einbringen von Schraube und Platte ist eine biologische Positionierung der Platte durch Einschieben unter den $\mathrm{m}$. vastus lateralis erleichtert.

Trotz der höheren Steifigkeit lässt sich bei fehlender medialer Abstützung keine Belastungsstabilität erreichen. Da es bei Anwendung der DCS nicht selten zu Pseudarthrosen und bei fehlender medialer Abstützung zu Varusfehlstellungen kam, spielt dieses Implantat heute keine führende Rolle mehr bei der Versorgung der subtrochantären Fraktur [2].

\section{Die dynamische Hüftschraube (DHS)}

Bei der DHS wird wie bei der DCS eine Schenkelhalsschraube mit einer an der Lateralseite befestigten Platte verbunden, allerdings verläuft die Schraube parallel zur Schenkelhalsachse. Bei pertrochantären Frakturverläufen kommt es durch die gewünschte Belastung der Fraktur zur Einstauchung und Impaktierung der Trümmerzone. Bei subtrochantären Frakturen ist das Implantat daher nur sinnvoll, wenn die Fraktur Ausläufer in die Trochanterregion hat, da nur dann das Prinzip des Implantates zur Wirkung kommt. Bei reinen subtrochantären Brüchen sollte sie dagegen nicht mehr verwandt werden.

\section{Der Gamma-Nagel}

Der Gamma-Nagel ist ein Hohlnagel für die Versorgung von Frakturen des proximalen Femurs. Im proximalen, verdickten Anteil des Nagels weist er ein Gleitloch für die Schenkelhalsschraube auf. Der Nagel kann distal einfach oder doppelt verriegelt werden. Zur Implantation wird ein Führungsspieß lateral der Trochanterspitze in den Markraum eingebracht, über den der Femur aufgebohrt wird. Der Nagel wird so weit eingeschlagen, dass die Schenkelhalsschraube im kaudalen Femurkopfdrittel zu liegen kommt. Die Schraube lässt sich im Nagel arretie- ren, so dass sie zwar gleiten, sich aber nicht mehr im Nagel drehen kann. Damit werden Rotationsfehler des Schenkelhalsfragmentes vermieden. Schenkelhalsschraube und Verriegelungsbolzen werden über ein Zielgerät eingebracht. Der Nagel ist in seiner Standardlänge und in überlangen Größen für die Versorgung von subtrochantären Frakturen erhältlich. In diesen Fällen erfolgt die distale Verriegelung in Freihandtechnik.

Die Vorteile der intramedullären Kraftträger gegenüber den Platten bei der Osteosynthese subtrochantärer Frakturen sind in vielen klinischen und biomechanischen Studien belegt. Götze et al. [3] konnten an Leichenfemora für den Gamma-Nagel eine Belastbarkeit von deutlich über der des Einbeinstandes nachweisen [3]. Zudem ist die Implantation in gedeckter Technik wesentlich leichter als bei Plattenosteosynthesen.

Mit diesem Implantat versorgte Frakturen sind daher in der Regel belastungsstabil. Ein weiterer Vorteil sind die minimal invasiven Zugänge. Der Nagel wird weit entfernt von der Frakturzone eingebracht, bei einfachen Frakturen muss die Frakturzone nicht eröffnet werden, die Fragmente werden nicht denudiert.

Da der Hohlnagel einen relativ großen Durchmesser hat, liegt er auch distal der Femurkortikalis eng an. Hierdurch entstehen am Nagelende Spannungsspitzen, die in der Anfangszeit häufig zu Femurschaftfrakturen distal der Nagelspitze geführt haben. Auch intraoperative Frakturen während der Insertion des Nagels wurden gesehen [1]. Diese Komplikationen werden verstärkt beobachtet, wenn distal - wie ursprünglich vorgesehen - doppelt verriegelt wird. Weiterhin können durch Fehlbohrungen der distalen Verriegelung Schwachstellen entstehen, die dann bei Bagatelltraumen zu Femurschaftfrakturen führen.

\section{Der proximale Femurnagel (PFN)}

Die Komplikationen des Gamma-Nagels führten zur Entwicklung des PFN. Es handelt sich um einen soliden Titannagel. Zur Implantation muss nur proximal aufgebohrt werden. Der Nagel ist dünner als der Gamma-Nagel, dadurch werden die Spannungsspitzen am Übergang zur Femurkortikalis minimiert. Die Kortikalis wird zudem weniger geschwächt, da auch dünnere Verriegelungsbolzen verwendet werden. Auch hier sind jedoch distale Fehlbohrungen zu vermeiden.
Wie der Gamma-Nagel ist auch der PFN für weit nach distal reichende Frakturen in Überlängen erhältlich, was ihn für die Versorgung von subtrochantären Frakturen zu einem idealen Implantat macht (Abb.3a u.b). Durch die Möglichkeit, proximal der Schenkelhalsschraube eine weitere Schraube als Antirotationsschraube einzubringen, lässt sich die Rotationsinstabilität bei Frakturen, die in die Trochanterregion einstrahlen, aufheben (Abb.4au.b). Diese hat eine besondere Bedeutung bei pertrochantären Frakturen. Auf diese zusätzliche Fixation kann verzichtet werden, wenn die Fraktur distal des Trochanter minor liegt und eine Rotationsstabilität im Schenkelhals nicht erzielt werden muss (Abb.5).

Die in vitro getestete mechanische Belastbarkeit des PFN liegt über der des Gamma-Nagels. Mit Standard- oder überlangem PFN versorgte subtrochantäre Frakturen sind deswegen als belastungsstabiler anzusehen.

\section{Der unaufgebohrte Femurnagel (UFN) mit Spiralklinge}

Die Implantationtechnik unterscheidet sich von den beiden zuvor beschriebenen Nägeln. Die Einschlagstelle ist die Fossa piriformis am Übergang vom Trochanter zum Schenkelhals. Zunächst wird ein Führungsdraht eingebracht, dessen korrekte Lage radiologisch kontrolliert wird. Nach Bestimmen der Nagellänge und Aufbohren wird auf das Nagelende eine spezielle Verriegelungshülse für die Klinge aufgesetzt. Die unterschiedlichen Hülsen lassen eine Klingenlage von $100^{\circ}, 110^{\circ}$ bzw. $120^{\circ}$ zu. Die Klinge muss vollständig im proximalen Fragment liegen, darf also von Frakturausläufern in die proximale Kortikales nicht tangiert werden. Dies gelingt meist bei einer eher horizontalen Implantation durch die $100^{\circ}$-Zielhülse. Probleme kann auch die Klingenplatzierung bereiten, da ein Antetorsionswinkel nicht vorgesehen ist und daher gelegentlich auch der Nagel im Schaft nach außen rotiert werden muss, um eine korrekte Position der Klinge im Schenkelhals zu erreichen.

Dieses Implantat kann bei subtrochantären Frakturen nur dann angewendet werden, wenn das Trochantermassiv intakt ist. Der UFN ist nicht Implantat der ersten Wahl bei subtrochantären Frakturen. 


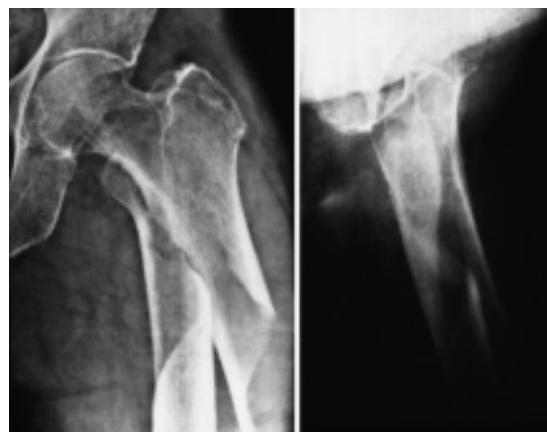

Abb.3a Subtrochantäre Fraktur.

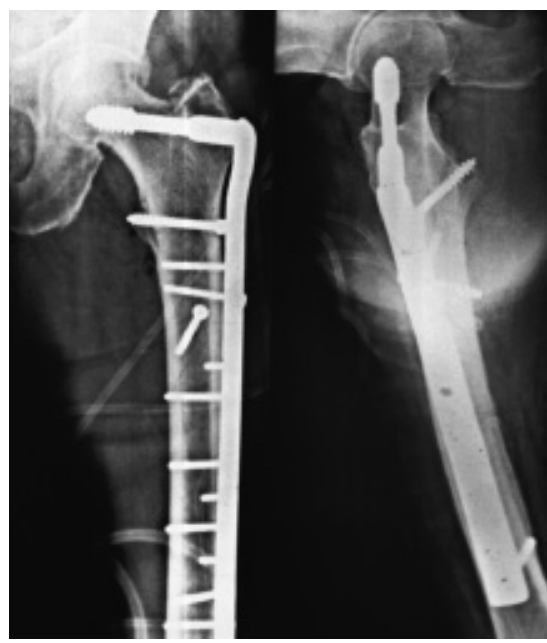

Abb. 3b Versorgung. Versorgung mit DCS in konventioneller Technik.
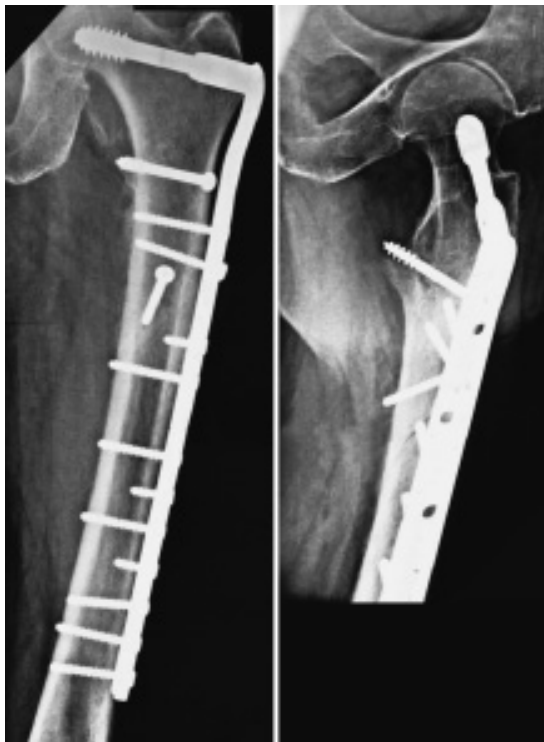

Abb.3c Ausheilung

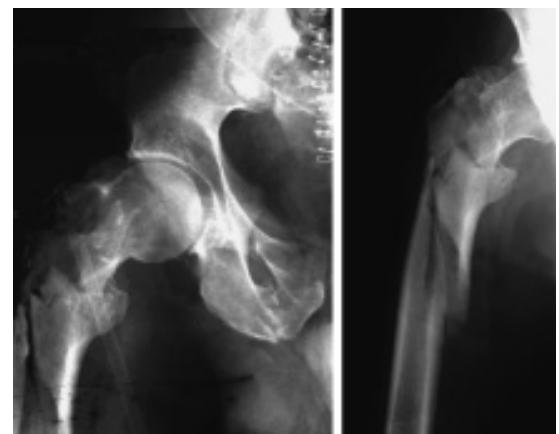

Abb.4a Subtrochantäre Fraktur.

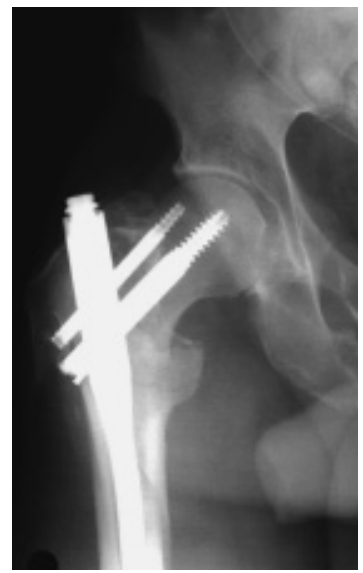

Abb. 4b Versorgung mit PFN. Ausheilung.

\section{Komplikationen}

Die Entwicklung in der Versorgung der subtrochantären Femurfraktur und die Vielzahl der möglichen Implantate zeigt, dass keines der Implantate frei von Komplikationen ist. Da bei Plattenosteosynthesen wie der Winkelplatte und der DCS vor allem bei ausgedehnter Freilegung der Fragmente gehäuft Pseudarthrosen und septische Komplikationen gesehen wurden [2], sollten diese Verfahren nur noch Ausnahmeindikationen vorbehalten bleiben.

Bei den in der Mehrzahl osteoporotischen Knochen der alten Patienten kann es aber trotz korrekter Implantation der Schenkelhalsschraube im kaudalen Schenkelhalsdrittel unter der Belastung zum Durchwandern der Schraube nach kranial kommen bis zum Durchwandern der Schraube durch den Hüftkopf.

Dies geschieht besonders dann, wenn zur Implantation der Schraube mehrere

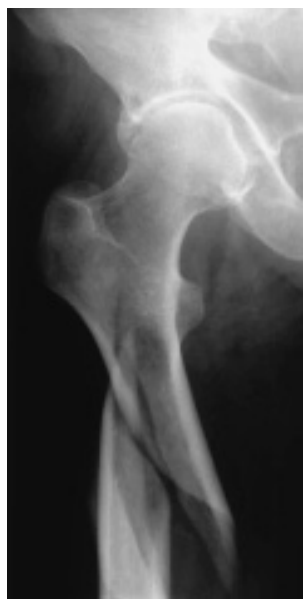

Abb. 5a Subtrochantäre Fraktur mit langem PFN

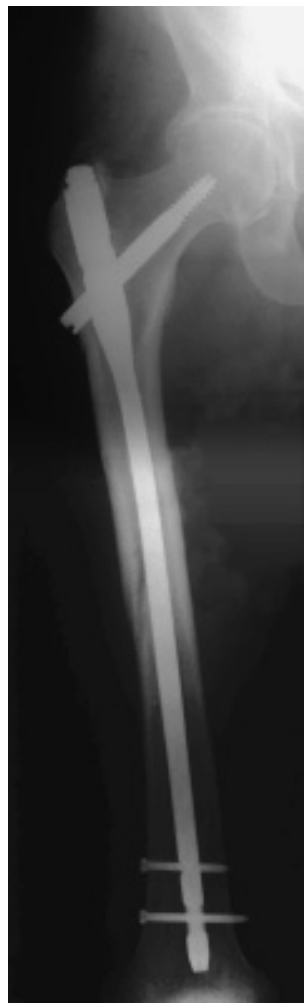

Abb. 5b Ausheilung mit langem PFN wurde. Durch einen zu nahen, am Adam-Bogen gesetzten Bohrvorgang kann außerdem die mediale Kortikales verletzt werden. Diese Komplikationen treffen auf alle Implantate mit einer Schenkelhalsschraube zu, also auch auf den Gamma-Nagel, den PFN und den UFN mit Spiralklinge.

Eine für den Gamma-Nagel typische Komplikation sind Frakturen distal des 
Nagelendes, die Folge einer erhöhten Spannung am Nagelende vor allem bei doppelter Verriegelung und eventueller Fehlbohrung sind [6]. Auch bei PFN und UFN werden solche Frakturen beschrieben, sie sind jedoch deutlich seltener.

Problematisch können die intramedullären Implantate bei Frakturenlinien sein, die durch die Nageleintrittstelle gehen. Beim Aufbohren - besonders beim sehr voluminösen proximalen Ende des Gamma-Nagels - wird die Trochanterzone weiter geschwächt, und beim Einbringen des Nagels kann es zu einer Sprengung des Schenkelhalsfragmentes und des Trochantermassivs kommen [7].

Eine besondere Komplikation des UFN mit Spiralklinge sind Rotationsfehler des Schenkelhalses; vor allem wenn die Fraktur nahe an die Schenkelschraube heranreicht. Deswegen besteht die Indikation zu diesem Implantat nur bei intaktem Trochantermassiv.

Allgemeine Komplikationen wie Blutungen, Hämatoserome, Infekte und Pseudarthrosen treffen auf alle Verfahren $\mathrm{zu}$ und sind abhängig vor allem von der Größe des unfallbedingten und operativ induzierten Weichteiltraumas sowie von der bei der Versorgung erreichten Stabilität.

\section{Schlussfolgerung}

Die Osteosynthesetechniken der hochgradig instabilen subtrochantären Frakturen haben in den letzten Jahren einen Wandel erfahren. Die extramedullären Plattenosteosynthesen werden heute seltener angewandt. Als Implantat der Wahl gelten heute die intramedullären Systeme wie PFN, Gamma-Nagel und vergleichbare Nagelsysteme. Bei weit nach distal reichenden subtrochantären Frakturen werden die überlangen Nägel bevorzugt. Vorteil der intramedullären Systeme sind in der Regel ein minimiertes Operationstrauma durch geschlossene Repositionstechniken sowie kürzere Operationszeiten. Der PFN hat eine extrem hohe mechanische Stabilität, begleitende Komplikationen wie Brüche am Nagelende oder intraoperative Schaftfrakturen werden seltener gesehen als bei dem Gamma-Nagel.

\section{Literatur}

1 Andreß H-J, Forkel H, Grubenwinkler M et al. Versorgung der per- bis subtrochantären $\mathrm{Fe}$ murfraktur durch Gamma-Nagel und modulare Hüftprothese. Unfallchir 103; 2000: 444-451

2 Bülhoff H, Dávid A, Ekkernkamp A. Versorgungsmöglichkeiten subtrochantärer Frakturen. Zentralbl Chir 120; 1995: 881/886

3 Götze B, Bonnaire F, Weise Ket al.. Belastbarkeit von Osteosynthesen bei instabilen perund subtrochantären Femurfrakturen: Ex- perimentelle Untersuchungen mit PFN Gamma-Nagel, DHS/Trochanterstabilisierungsplatte, $95^{\circ}$-Condylenplatte und UFN Spiralklinge. Akt Traumatol 28; 1998: 197-204

${ }^{4}$ Kinast C, Bolhofner BR, Mast JW et al. Subtrochanteric Fractures of the Femur. Clin Orthop 238; 1989: $122-130$

5 Richter D, Hahn MP. Trochantäre Frakturen. In: Szyszkowitz R, Muhr G, Trentz O: Operative Therapie der Frakturen und Luxationen. Ecomed, Landsberg/Lech 2001: 1-60

6 Sailer R, Ulmer H, Hrubesch R et al. Operative Stabilisierung von per- und subtrochantären Oberschenkelfrakturen mit dem Gamma-Nagel. Chirurg 71; 2000: 1380-1384

7 Wagner S, Rüter A. Per- und subtrochantäre Femurfrakturen. Unfallchir 102; 1999: 206 222

8 Weise K, Schwab E. Intramedulläre Kraftträger zur Versorgung der per- und subtrochantären Femurfraktur. Chirurg 72; 2001 : $1277-1282$

9 Wiss D, Brien WW. Subtrochanteric Fractures of the Femur. Clin Orthop 283; 1992: $231-236$

\section{Dr. med Thomas Strohecker}

Oberarzt

Dr. med Axel Pommer

Leitender Oberarzt

Prof. Dr. med. Andreas Dávid

Klinikdirektor

Klinik für Unfall- und Wieder-

herstellungschirurgie

Heusnerstr. 40

D-42283 Wuppertal 